\title{
Expression of Matrix Metalloproteinase-9 in Tongue Squamous Cell Carcinoma Correlates with Lymph Node Metastasis- A Clinicopathologic Study
}

\author{
Yuanyuan Zhang', ${ }^{1,}$, Yoshinori Jinbu', Hiroto Itoh ${ }^{1}$, Mikio Kusama ${ }^{1}$, Keiichi Tsukinoki ${ }^{3}$, Motoshi Kikuchi ${ }^{4}$ \\ and Takashi Yashiro ${ }^{4}$ \\ ${ }^{1}$ Department of Dentistry, Oral and Maxillofacial Surgery, ${ }^{4}$ Department of Histology and Anatomy, Jichi Medical School, Tochigi, \\ Japan \\ ${ }^{2}$ Department of Oral Surgery, The Second Affiliated Hospital, China Medical University, Shenyang, China \\ ${ }^{3}$ Department of Oral Pathology, Kanagawa Dental College, Kanagawa, Japan
}

Zhang Y, Jinbu Y, Itoh H, Kusama M, Tsukinoki K, Kikuchi M and Yashiro T. Expression of matrix metalloproteinase-9 in tongue squamous cell carcinoma correlates with lymph node metastasis-a clinicopathologic study. Oral Med Pathol 2005; 89-94, ISSN 1342-0984

Invasion and cervical lymph node metastasis are two factors governing the poor prognosis of patients with tongue cancer. Matrix metalloproteinase-9 (MMP-9) has been implicated in invasion and lymph node metastasis of several tumor types. However, the relationship between MMP-9 expression and clinicopathologic characteristics in human tongue cancer has little been reported. This study included 48 patients with tongue cancer, of which 18 cases showed lymph node metastasis. The relationship between MMP-9 expression and the mode of invasion and nodal involvement was immunohistochemically examined. MMP-9 overexpression was identified in 31 cases $(64.6 \%)$. Frequent expression of MMP-9 was statistically significant $(p<0.05)$ in the cases with lymph node metastasis or advanced stage. MMP-9 may play an important role in the invasion and metastasis of tongue cancer. Thus, analysis of MMP-9 production may be useful for the evaluation of malignant potential in human tongue cancer.

Key Words: matrix metalloproteinase-9, tongue cancer, invasion, metastasis

Correspondence: Yoshinori Jinbu, Department of Dentistry, Oral and Maxillofacial Surgery, Jichi Medical School, Yakushiji 3311-1, Minamikawachi-machi, Kawachi-gun, Tochigi 329-0431, Japan Phone: +81-285-58-7390, Fax: +81-285-44-8669, E-mail: jinbu@jichi.ac.jp

\section{Introduction}

Cure rate of oral cancer with available treatment modalities has not improved significantly over the last few years (1). Additional therapeutic measures, such as chemotherapy and radiotherapy, either pre- or post-operatively, are not suitable for every patient with tongue cancer because of their hazardous side-effects (2). Therefore, it is important to develop new diagnostic markers to identify patients at high risk for cancer and to consider more intensive treatment.

Invasion and cervical lymph node metastasis are two factors governing the poor prognosis of patients with tongue cancer $(2,3)$. The process of tumor cell invasion through the extracellular basement membrane and stroma requires a number of steps including binding, penetration and migration through the underlying basement membrane (4). The degradation of extracellular matrix is without doubt a key element in tumor invasion and metastasis.
Matrix metalloproteinases (MMPs) are a family of zinc-containing proteases, which collectively are capable of degradation of all components of the extracellular matrix (5). The $92 \mathrm{kDa}$ type IV collagenase (matrix metalloproteinase-9, MMP-9) that degrades type IV collagen consisting basement membranes is implicated in the invasion and lymph node metastasis of several tumor types (6-10). However, there have been few studies on the expression of MMP-9 and the correlation with the clinicopathologic characteristics in human tongue cancer.

In this study we used immunohistochemical techniques to evaluate the relationship between MMP-9 expression and the metastatic potential of tongue cancer.

\section{Materials and methods}

Specimens

Oral biopsy specimens of 48 cases of tongue cancer and 5 cases of normal tongue mucosa were obtained from 
China Medical University First Hospital. Clinicopathologic information, such as age, gender, and location, was reviewed from the clinical sheets and pathologic reports. None of the patients had received chemotherapy or radiotherapy before surgical treatment. A qualified oral pathologist confirmed the presence of lymph node metastasis, differentiation and the mode of invasion (MI). The study was approved by the Medical Ethics Committee of China Medical University.

\section{Determination of invasiveness}

The degree of invasion was divided into four types from grade 1 to grade 4 according to the method reported previously (11).

Grade 1: nodule type growth of tumor mass into the surrounding tissue with distinct border; Grade 2: cord type growth of tumor mass into the surrounding tissue with distinct border; Grade 3: cord type growth of tumor mass into the surrounding tissue with indistinct border; Grade 4c: a cord-like type of diffuse invasion in which tumor cells, invade deeply as a cord or round-shaped microtumor nest; Grade 4d: a wide spread type of diffuse invasion in which tumor cells, either singly or a few at a time, invade diffusely into the deepest portion of the tissue.

\section{Immunohistochemistry}

Paraffin sections (5 $\mu \mathrm{m}$ ) were deparaffinized and dehydrated through graded ethanol, and endogenous peroxidase activity was blocked by $0.3 \%$ hydrogen peroxide in methanol for 30 minutes after they were autoclaved in $0.01 \mathrm{M}$ citrate buffer $(\mathrm{pH}=6.0)$ at $121^{\circ} \mathrm{C}$ for 10 minutes to retrieve the antigen. Before the application of primary antibody (mouse anti-human monoclonal antibodies to MMP-9, Daiichi Fine Chemical Co. Ltd., Takaoka, Japan), the sections were saturated in $2 \%$ normal goat serum for 20 minutes and then incubated with primary antibody $(1 \times 200)$ in a moist chamber at $30^{\circ} \mathrm{C}$ overnight. Sections were incubated in biotinylated goat anti-mouse antibody ( $1 \times 133$, Vector Laboratory, Burlingame, CA, USA) for 30 minutes and then with $\mathrm{ABC}$ peroxidase (Vector Laboratory, Burlingame, CA, USA) for 30 minutes. Immunohistochemical reactions were visualized by freshly prepared 3,3'-diaminobenzidine tetrahydrochloride (Wako Pure Chemical Industries, Osaka, Japan) in 0.05M TBS containing $0.005 \%$ hydrogen peroxide. After counterstaining with hematoxylin for 5 minutes, the sections were observed under a light microscope. For positive controls, human colon adenocarcinoma was used. Negative control was performed by substituting the primary antibody with normal mouse serum.

The degree of staining for MMP-9 in tumor cells was evaluated by an oral pathologist, to whom the clinical data was unknown. All the cases were classified into groups based on the degree of MMP-9 staining as de-
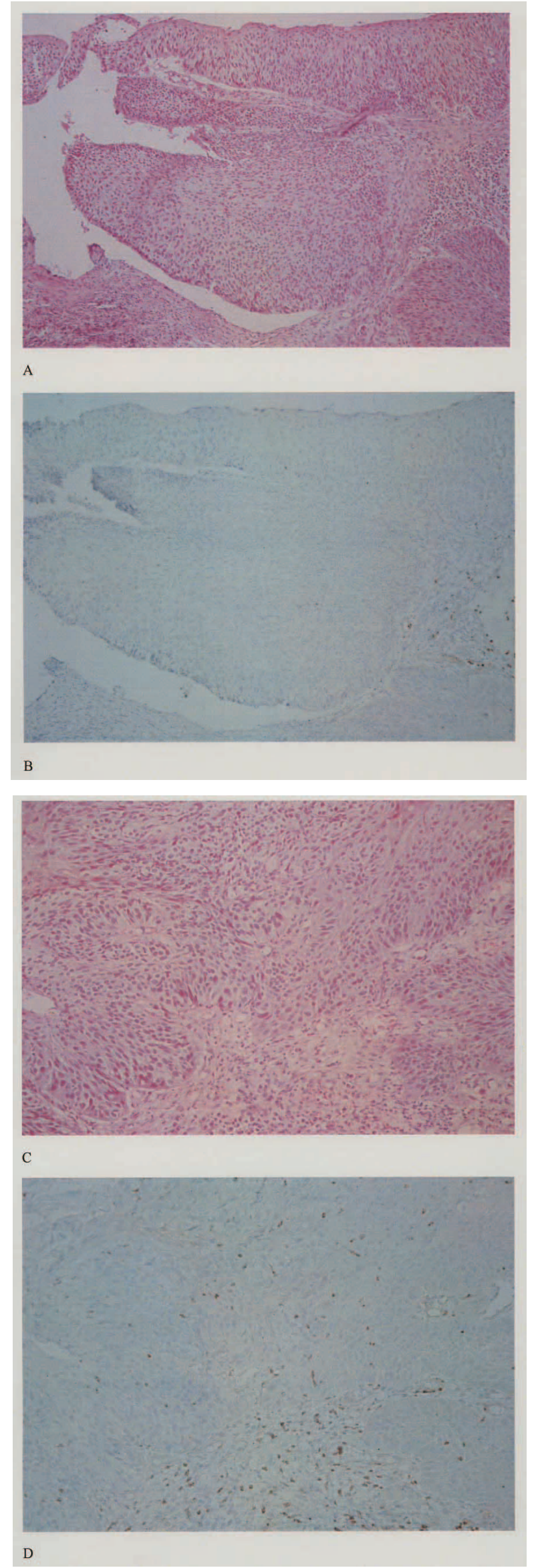


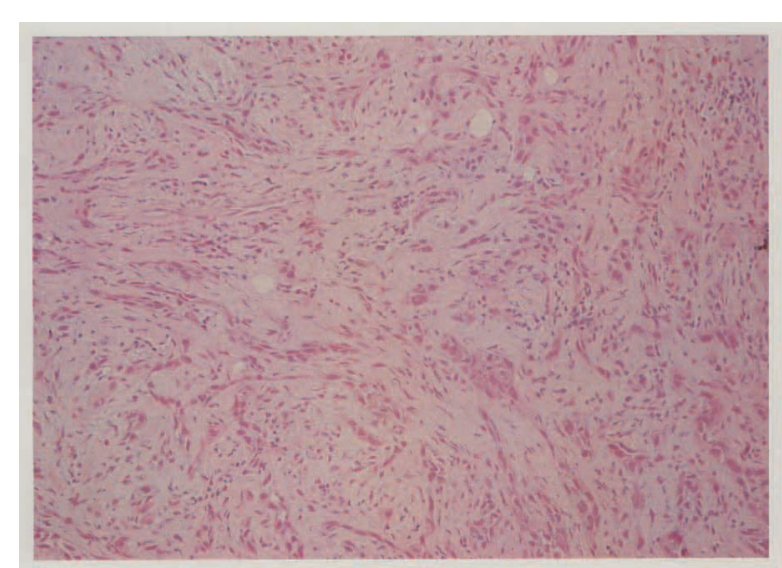

E
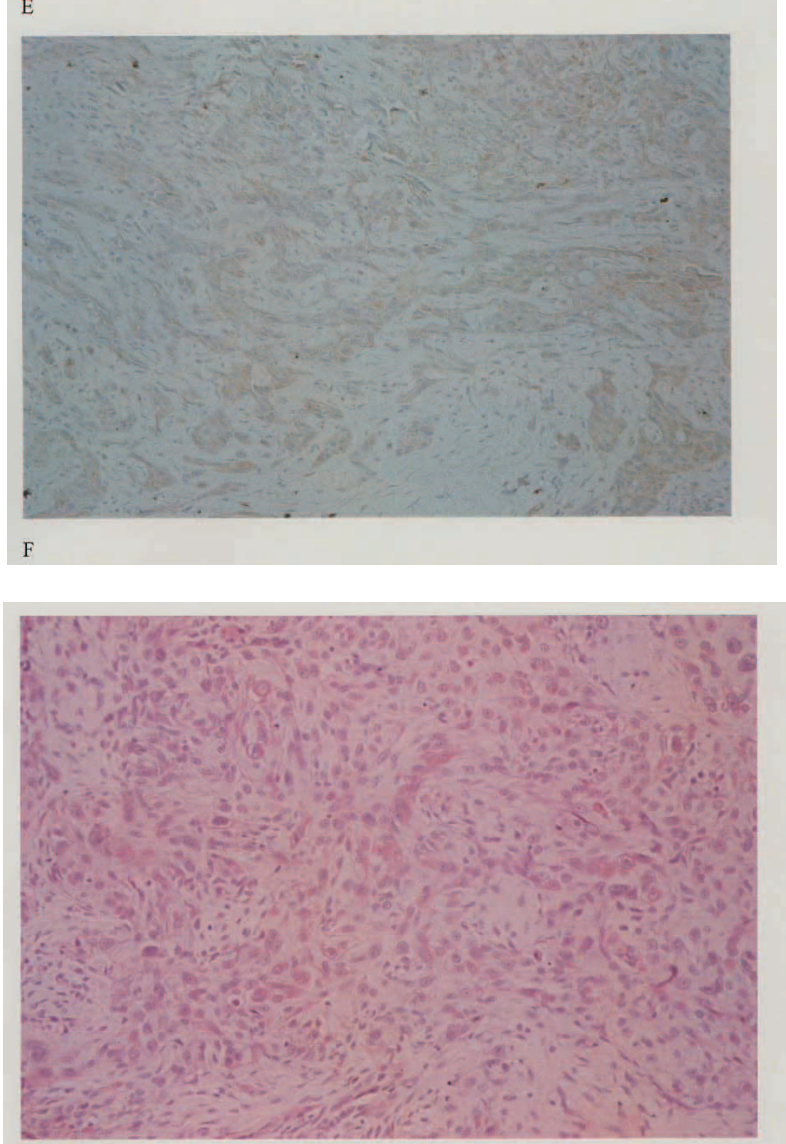

G

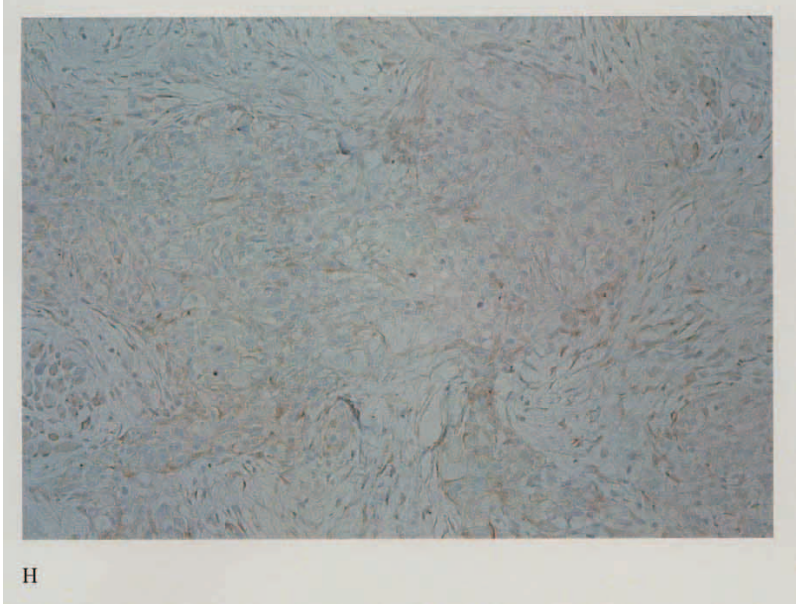

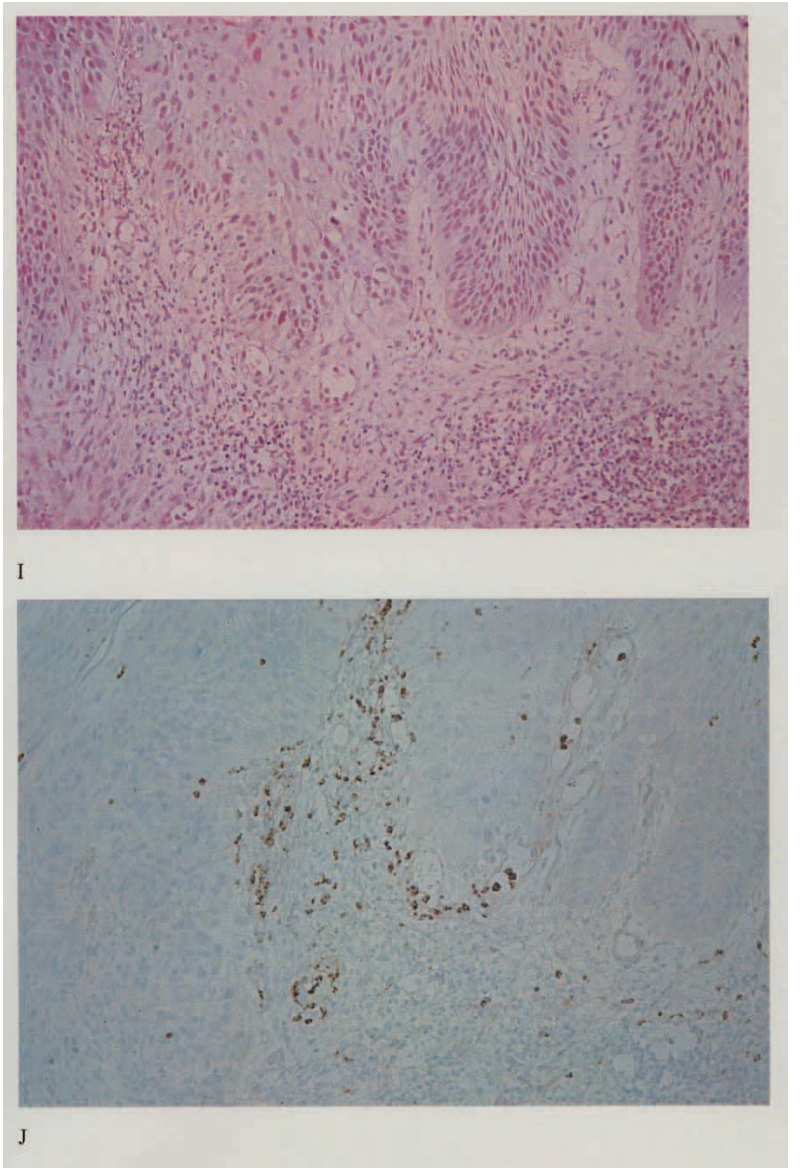

Figs. A to H: Representative pictures to show the mode of invasion (HE) and expression of MMP-9 in tumor cell (Immunohistochemical staining)

A: Mode of invasion 1; tumor mass of nodule type grow into the surrounding tissue with distinct border.

B: 0 ; tumor cells show almost completely negative staining of MMP-9.

C: Mode of invasion 1; tumor mass of nodule type grow into the surrounding tissue with distinct border.

D: $1+$; the tumor shows positive staining in some of the tumor cells.

E: Mode of invasion 4C; tumor cells invade deeply into the surrounding tissue as a cord-like type.

F: $3+$; tumor cells show extensive staining throughout the tumor mass.

G: Mode of invasion 4D; tumor cells invade diffusely into the deepest portion of the tissue.

$\mathrm{H}: 3+$; tumor cells show extensive staining of MMP-9. (Original magnification: A and B $\times 200$; C- $\times 400$ )

Figs. I and J: Expression of MMP-9 in stromal cells.

I: Mode of invasion 1; tumor mass of nodule type grow into the surrounding tissue with distinct border.

$\mathrm{J}$ : The stained cells in stroma included inflammatory cells and fibroblast cells, which concentrated at the interface between stroma and tumor components and surrounded the cancer nest. (Immunohistochemical staining) (Original magnification: I and $\mathrm{J} \times 400$ ) 
scribed previously (12): 0 , negative staining of tumor cells; $1+$, low expression ( $<10 \%$ positive tumor cells); $2+$, moderate expression (10-50\% positive tumor cells); $3+$, diffuse expression ( $>50 \%$ positive tumor cells). $2+$ and $3+$ were accepted as positive in this experiment.

\section{Statistical analysis}

Statistical analysis was performed using Chisquare test, and $p$ values less than 0.05 were considered to be significant.

\section{Results}

Immunohistochemical expression of MMP-9

No obvious immunostaining of MMP-9 was observed in normal tongue mucosa. In the specimens from 48 patients with tongue cancer, neoplastic cells, inflammatory cells and fibroblast cells variously expressed MMP-9. Positive expression of MMP-9 in tumor cells was detected in 31 cases $(64.6 \%)$.

MMP-9 was predominantly immunolocalized in the tumor cells, in which positive enzyme reactions were observed in the membrane and cytoplasm of the tumor cells (Figs. A-J). The stained cells in stroma included inflammatory cells and fibroblast cells, which concentrated at the interface between stroma and tumor components and surrounded the cancer nest (Figs. I and J).

MMP-9 expression of and clinicopathologic characteristics

Table 1 summarizes findings for the relationships between MMP-9 expression and clinicopathologic parameters of tongue cancer.

The percentages of positive cases increased with the advancement of clinical stages; a statistically significant difference was observed between the advanced cases (stage III and IV) $(20 / 24,83.3 \%)$ and those in the early stage (stage I and II) $(11 / 24,45.8 \%)(p<0.01)$. Positive expression of MMP-9 was also detected more frequently in the cases with nodal involvement $(15 / 18,83.3 \%)$ than in the cases without metastasis $(16 / 30,53.3 \%)(p<0.05)$.

Table1: Relationship between MMP-9 expression and clinicopathologic characteristics of tongue cancer

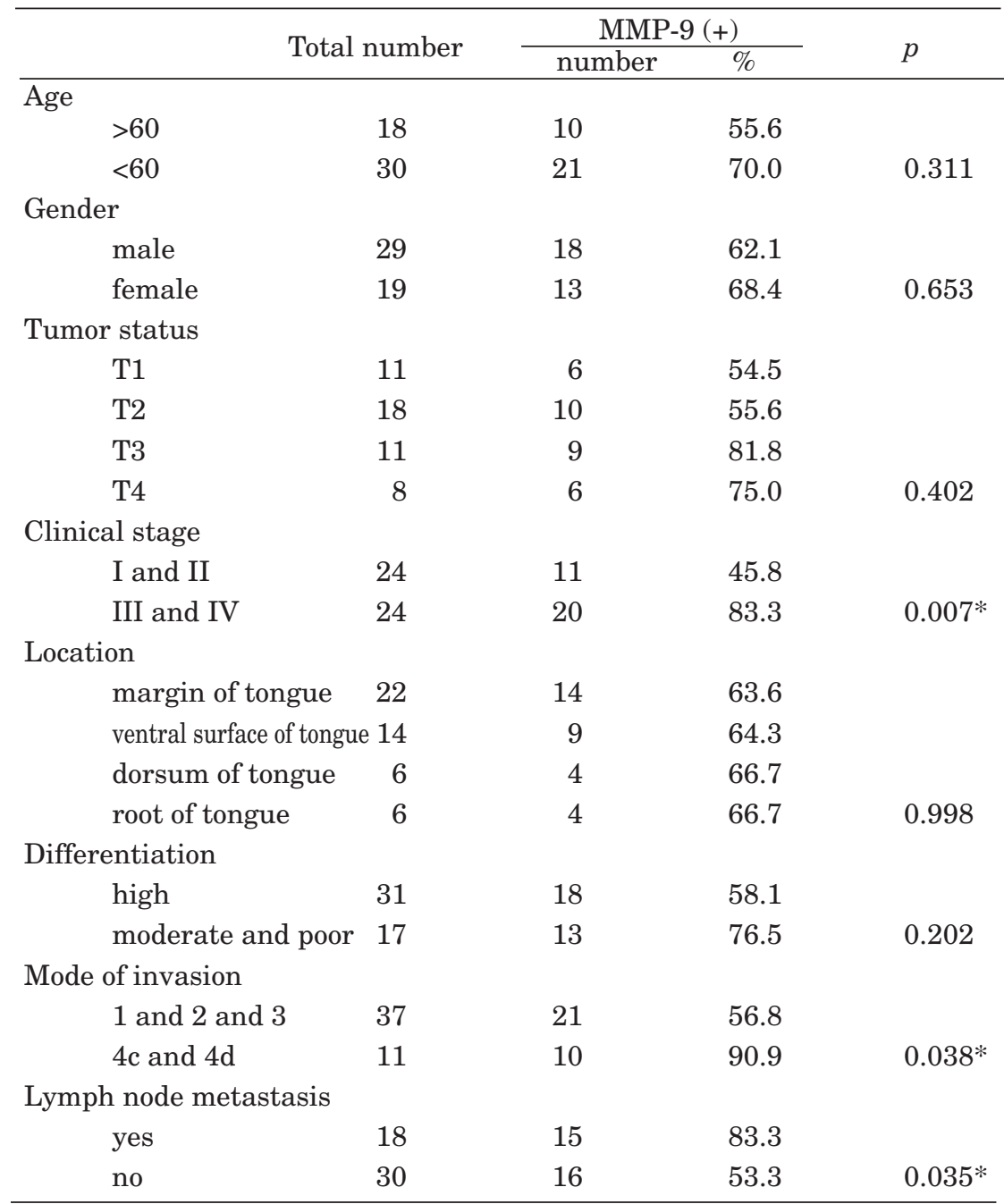

Statistical analysis was performed by Chi-square test.

$* p<0.05$ was considered statistically significant. 
A higher percentage of positive cases was observed in the specimens with advanced mode of invasion (MI 4c and 4d) (10/11, 90.9\%) compared with less invasive cases (MI $1,2$ and 3$)(21 / 37,56.8 \%)(p<0.05)$.

No statistical significance was found between the groups divided according to age, gender, tumor status, location and differentiation.

\section{Discussion}

Matrix metalloproteinases have been reported to play direct roles in other cellular activities, such as differentiation, proliferation, invasion and metastasis. A major function of MMP-9 in invasion and metastasis is to degrade type IV collagen in basement membranes and facilitate the breakdown of extracellular matrix to prompt the invasion and metastasis of tumors (13). Another important step of tumor progression in which MMP-9 has been implicated is angiogenesis. MMP-9 may be an important regulator of angiogenesis that cooperates with other factors, such as NO and VEGF, and supports the process of neovascularization, and thus, prompts the invasion and metastasis (12). In the present study, MMP9-positive expression was observed more frequently in cases with lymphatic metastasis or advanced clinical stage, which indicates MMP-9 may play an important role in invasion and metastasis in human tongue cancer.

The concept that tumor stroma may favor tumorigenesis is now well accepted $(14,15)$. Recent studies indicate that MMPs produced by inflammatory cells may make a relevant contribution to squamous cell carcinogenesis in a transgenic mouse model (16). In our study, MMP-9 was expressed largely in tumor cells but also in the adjacent stromal cells and inflammatory cells. Our study confirms previous findings (12) and provides further evidence that both tumor cells and stromal cells are the sources of MMP-9 in human tongue cancer. In the present study, the expression of MMP-9 by inflammatory cells and fibroblast cells was found at the interface between stroma and tumor components. Similar results have been reported in other tumor types, including skin (17) and colorectal tumor (18). The dynamic host-tumor interactions may modulate the level of MMP-9 and influence the progression of tongue cancer (12). However, very few patients showed MMP-9 positive cells in stroma and most positive cells were inflammatory cells. Therefore, we evaluated MMP-9 positive tumor cells in this study.

It is generally accepted that cervical lymph node metastasis, advanced clinical stage, and advanced mode of invasion in patients with tongue cancer indicates a poor prognosis. Compared to other types of oral cancer, tongue cancer metastasizes much more easily to cervical lymph nodes primarily because of the free mobility and the rich lymphatics in the submucosa of the tongue (19). Many studies have shown that tumor-cell dissemination to cervical lymph nodes occurs before successful treatment of the primary tumor (20). High expression of MMP-9 correlating with positive cervical lymph node metastasis, advanced clinical stage and high invasion indicates that the evaluation of MMP-9 could be valuable in identifying patients at high risk for prognosis who should be treated as more advanced cases.

\section{References}

1. Silverman S Jr. Demographics and occurrence of oral and pharyngeal cancers: The outcomes, the trends, the challenge. J Am Dent Assoc 2001; 132: 7-11.

2. Unal OF, Ayhan A and Hosal AS. Prognostic value of p53 expression and histopathological parameters in squamous cell carcinoma of oral tongue. J Laryngol Otol 1999; 113: 446-50.

3. Kirita T, Okabe S, Izumo T, et al. Risk factors for the postoperative local recurrence of tongue carcinoma. J Oral Maxillofac Surg 1994; 52: 149-54.

4. Hong SD, Hong SP, Lee JI, et al. Expression of matrix metalloproteinase-2 and -9 in oral squamous cell carcinomas with regard to the metastatic potential. Oral Oncol 2000; 36: 207-13.

5. Curran S and Murray GI. Matrix metalloproteinases in tumour invasion and metastasis. J Pathol 1999; 189: 300-8.

6. Tanioka Y, Yoshida T, Yagawa T, et al. Matrix metalloproteinase- 7 and matrix metalloproteinase- 9 are associated with unfavourable prognosis in superficial oesophageal cancer. Br J Cancer 2003; 89: 2116-21.

7. Kurahara S, Shinohara M, Ikebe T, et al. Expression of MMPs, MT-MMP, and TIMPs in squamous cell carcinoma of the oral cavity: correlations with tumor invasion and metastasis. Head Neck 1999; 21: 627-38.

8. Kabashima A, Maehara Y, Kakeji Y, et al. Clinicopathological features and overexpression of matrix metalloproteinases in intramucosal gastric carcinoma with lymph node metastasis. Clin Cancer Res 2000; 6: 3581-4.

9. Horikawa T, Yoshizaki T, Sheen TS, et al. Association of latent membrane protein 1 and matrix metalloproteinase 9 with metastasis in nasopharyngeal carcinoma. Cancer 2000; 89: $715-23$.

10. Maeta H, Ohgi S and Terada T. Protein expression of matrix metalloproteinases 2 and 9 and tissue inhibitors of metalloproteinase 1 and 2 in papillary thyroid carcinomas. Virchows Arch 2001; 438: 121-8.

11. Yamamoto E, Kohama G, Sunakawa H, et al. Mode of invasion, bleomycin sensitivity, and clinical course in squamous cell carcinoma of the oral cavity. Cancer 1983; 51: 2175-80.

12. Franchi A, Santucci M, masini E, et al. Expression of matrix metalloproteinase 1 , matrix metalloproteinase 2 , and matrix metalloproteinase 9 in carcinoma of the head and neck. Cancer 2002; 95: 1902-10.

13. Ranuncolo SM, Matos E, Loria D, et al. Circulating 92kilodalton matrix metalloproteinase (MMP-9) activity is enhanced in the euglobulin plasma fraction of head and neck squamous cell carcinoma. Cancer 2002; 94:1483-91. 
14. Stetler-Stevenson WG, Hewitt R and Corcoran M. Matrix metalloproteinases and tumor invasion: from correlation and causality to the clinic. Semin Cancer Biol 1996; 7: 147-54.

15. MacDougall JR and Matrisian LM. Contributions of tumor and stromal matrix metalloproteinases to tumor progression, invasion and metastasis. Cancer Metastasis Rev 1995; 14: 351-62.

16. Coussens LM, Tinkle CL, Hanahan D, et al. MMP-9 supplied by bone marrow-derived cells contributes to skin carcinogenesis. Cell 2000; 103: 481-90.

17. Pyke C, Ralfkiaer E, Huhtala P, et al. Localization of messenger RNA for Mr 72,000 and 92,000 type IV collagenases in human skin cancers by in situ hybridization. Cancer Res 1992; 52: 1336-41.

18. Pyke C, Ralfkiaer E, Tryggvason K, et al. Messenger RNA for two type IV collagenases is located in stromal cells in human colon cancer. Am J Pathol 1993; 142: 359-65.

19. Cummings CW, Fredrickson JM, Harker LA, et al. Malignant neoplasms of the oral cavity. In: Pramod K, ed. Otolaryngology Head and Neck Surgery. Mosby-year Book, Missouri. 1998; 1424-5.

20. Kurokawa H, Yamashita Y, Takeda S, et al. Risk factors for late cervical lymph node metastases in patients with stage I or II carcinoma of the tongue. Head Neck 2002; 24: 731-6.

(Accepted for publication April 27, 2005 ) 